\title{
Ibuprofen Release from Poly(ethyl cyanoacrylate) Nanoparticles Prepared by Semicontinuous Heterophase Polymerization
}

\author{
J. A. Balleño, ${ }^{1}$ A. P. Mendizábal-Ruiz ${ }^{\mathbb{D}},{ }^{1}$ H. Saade $\mathbb{D},{ }^{2}$ R. Díaz de León-Gómez $\mathbb{D}{ }^{2}{ }^{2}$ \\ E. Mendizábal $\mathbb{D},{ }^{1}$ N. Rios-Donato, ${ }^{1}$ and R. G. López $\mathbb{D}^{2}$ \\ ${ }^{1}$ Centro Universitario de Ciencias Exactas e Ingenierías, Universidad de Guadalajara, Blvd. M. García-Barragán, No. 1451, \\ 44430 Guadalajara, JAL, Mexico \\ ${ }^{2}$ Departamento de Procesos de Polimerización, Centro de Investigación en Química Aplicada, Blvd. Enrique Reyna, No. 140, \\ 25294 Saltillo, COAH, Mexico
}

Correspondence should be addressed to R. G. López; guillermo.lopez@ciqa.edu.mx

Received 22 August 2017; Revised 9 January 2018; Accepted 2 April 2018; Published 21 May 2018

Academic Editor: Hui-Yun Zhou

Copyright (C) 2018 J. A. Balleño et al. This is an open access article distributed under the Creative Commons Attribution License, which permits unrestricted use, distribution, and reproduction in any medium, provided the original work is properly cited.

\begin{abstract}
Ibuprofen-loaded poly(ethyl cyanoacrylate) nanoparticles were prepared by semicontinuous heterophase polymerization of ethyl cyanoacrylate in the presence of ibuprofen; different surfactant concentration, $\mathrm{pH}$, and temperature were used. Particle size was measured by quasi-light scattering and transmission electron microscopy, while the amount of drug released was determined by UV spectroscopy. Nanoparticles with diameters between 10 and $58 \mathrm{~nm}$, loaded with ibuprofen, were obtained. The smallest particles and the higher drug loading were obtained at the highest $\mathrm{pH}$ tested. The analysis of the release data showed that the drug release profiles correspond to the Weibull model. Moreover, it was found that most of the ibuprofen is released within the first 80-120 min; initially the release rate is slow, but then it increases to finally decrease. This behavior contrasts with the reported burst of drug concentration in the plasma after oral administration of IB.
\end{abstract}

\section{Introduction}

Drug-loaded nanostructures are a subject of increasing interest for a large number of research groups around the world. Some of their potential advantages for treatment of various diseases include controlled release, greater selectivity, longer times in the blood stream, decrease of side effects, and attenuation of multidrug resistance, which finally leads to an increase in the efficacy of the drugs [1-4]. Among the nanostructures used for drug loading, liposomes, inorganic particles, polymeric micelles, quantum dots, polysaccharide particles, dendrimers, and polymeric particles can be included $[5,6]$. Each of these types of nanostructures provides advantages and drawbacks for drug administration; however, polymeric nanoparticles perhaps are the ones with greater potential [7]. It is well known that particles with diameters less than $5.5 \mathrm{~nm}$ are quickly removed by the kidneys [8], while those larger than $100 \mathrm{~nm}$ are taken off from the blood stream by the liver and the spleen [3, 9-12]. Moreover, it is also known that there is an inverse relationship between the nanostructure size and its circulation time in the blood stream. Thus, the particle size required to reach the longest circulation times in the blood stream is just above $5.5 \mathrm{~nm}$ in diameter. However, plenty of papers in the specialized literature dealing with the preparation of drug-loaded polymer nanoparticles [7] report the obtaining of structures with diameters greater than $50 \mathrm{~nm}$.

Recently, our group reported the preparation of biocompatible and biodegradable poly(ethyl cyanoacrylate) (PECA) nanoparticles with $30 \mathrm{~nm}$ in mean diameter, loaded with rifampicin [13]. These small sizes were obtained as a result of using a polymerization method developed by our group, called semicontinuous heterophase polymerization (SCHP) [14].

In this method, nanoparticles are prepared by a polymerization in which the monomer is added at very slow dosing rates over a surfactant containing solution, which compels the monomer to be below its saturation concentration inside the particles. In turn, this slows down particle growth, leading to a large number of smaller particles in accordance with 
the emulsion polymerization theory $[15,16]$. A more detailed explanation of this effect has already been reported elsewhere by our group $[17,18]$.

Taking into account the importance of ibuprofen (IB), which is one of the most widely used nonsteroidal antiinflammatories in the world, this drug was chosen to be loaded onto PECA nanoparticles prepared by SCHP. In contrast with our previous report on preparation of drug-loaded PECA nanoparticles, in which rifampicin was loaded once the particles were obtained [13], in this document we report the preparation of IB-loaded PECA nanoparticles by carrying out the SCHP in the presence of the drug, varying the surfactant concentration, $\mathrm{pH}$, and temperature. The IB release from the PECA nanoparticles was followed as a function of time, and the experimental data were fitted using the Higuchi, Korsmeyer-Peppas, Hixson, and Weibull models.

\section{Materials and Methods}

2.1. Materials. The monomer, ethyl-2-cyanoacrylate (ECA), the surfactant Tween 80 (TW 80), and the drug, ibuprofen sodium salt $(\geq 98 \%)$, were purchased from Sigma-Aldrich ${ }^{\circledR}$ and used as received. The ECA was stored under refrigeration $\left(-6^{\circ} \mathrm{C}\right)$ until used in the polymerization reaction. Bidistilled and deionized water was employed.

2.2. Polymerizations and Purification. Polymerizations were carried out in a $50 \mathrm{~mL}$ two-necked jacketed reactor; one of the inlets was used to dose the monomer with a $1 \mathrm{~mL}$ syringe (BD Ultra-Fine ${ }^{\circledR}$ for insulin U-100 X $13 \mathrm{~mm}$ ) and the other for mechanical stirring (400 RPM). A micellar solution was prepared using TW 80 and water. Ibuprofen has a very low solubility in aqueous solutions $(0.0001 \mathrm{~g} / \mathrm{mL})$, so if it is added to the micellar solution, its dispersion is not adequate for good incorporation into the micelles. For this reason IB as salt, which is very water-soluble, was added to the micellar solution. Then the acidic $\mathrm{pH}$ of the medium was adjusted using an aqueous solution of $\mathrm{HCl} 1 \mathrm{~N}$, whereupon the $\mathrm{IB}$ becomes lipophilic, allowing it to be incorporated into the micelles. The reactor was cooled to the reaction temperature before initiating monomer addition. The monomer was added at a constant flow in a two-hour period. The reaction temperature was maintained constant using a recirculating bath with a digital temperature controller (Intertek, model AD7LR-20). After completing the ECA addition, the reaction was allowed to proceed for one hour more. A portion of the resulting latex was purified by dialysis at a $\mathrm{pH}$ of 7.5 to remove surfactant and the ibuprofen not trapped in the particles. The washing water was withdrawn from the dialysis system each 24 hours and distilled water was added to continue the purification process. The removed water was analyzed by UV spectroscopy (Thermo Scientific, Model Genesys 10) to determine ibuprofen and surfactant presence. Dialysis continued until the washing solution did not contain ibuprofen and surfactant. To determine the IB content in the particles, an aliquot of the purified latex was taken, diluted by a factor of $1: 20$, and neutralized with an aqueous solution of $\mathrm{NaOH}$ to solubilize the ibuprofen. The IB content was determined by UV/Vis spectroscopy (Thermo
Scientific, Model Genesys 10) at a wavelength of $222 \mathrm{~nm}$. The IB content determinations were carried out in quintuplicate. The nanoparticles were allowed to settle and the supernatant liquid was removed; then the particles were dried in an oven (NAPCO Vacuum Oven Model 5831) at a temperature of $35^{\circ} \mathrm{C}$ and vacuum (pressure of 25 in $\mathrm{Hg}$ ) until constant weight. Monomer conversion was determined by gravimetry.

\subsection{Characterization}

2.3.1. FTIR Spectroscopy. A Fourier Transform Infrared Spectrophotometer FTIR (Nicolet 6700) was employed to obtain the spectra using the Attenuated Total Reflectance (ATR) Smart Orbit accessory.

2.3.2. Molecular Weight. Average molecular weights were determined in a Breeze 2 HPLC system (Waters) calibrated with polystyrene standards (Polyscience). For this measurement, $50 \mathrm{mg}$ of purified PECA was dissolved in $5 \mathrm{~mL}$ of HPLC-grade tetrahydrofuran; then the solution was filtered and injected into the apparatus. The equipment was operated at a flow of $1 \mathrm{~mL} / \mathrm{min}$ and a temperature of $30^{\circ} \mathrm{C}$.

2.3.3. Particle Size. Particle size and shape were determined by TEM in a JEOL JEM 2100 equipment; for this, the PECA nanoparticles were dispersed in distilled water using an ultrasonicator; then a drop of the dispersion was deposited on a copper grid, where the solvent was allowed to evaporate.

Quasi-elastic light scattering (QLS) was also employed to determine the particle size of the nanoparticles. Intensity correlation function measurements were carried out using a Brookhaven BI-9000AT 522 digital channel correlator, equipped with a water-cooled argon-ion laser, operated at $514.5 \mathrm{~nm}$, as a light source. To eliminate multiple scattering and particle interactions, the latexes samples were diluted 50 times with water. The measurements were made at $25^{\circ} \mathrm{C}$ and $\mathrm{pH}$ of 7.0. Samples measurement was made in triplicate. The particle size was obtained by CONTIN analysis.

2.4. In Vitro Release IB Study. To carry out the drug release experiments, $0.05 \mathrm{~g}$ of IB-loaded PECA was added to $10 \mathrm{~mL}$ of an aqueous solution at $\mathrm{pH}$ of 7.5. This $\mathrm{pH}$ was chosen because the $\mathrm{pH}$ of the blood is in the range of 7.44-7.6; besides ibuprofen presents appreciable water solubility at $\mathrm{pH}$ $>7.0[19,20]$. The suspension was maintained under constant stirring for 5 hours. Aliquots of the solution were taken every 30 minutes, diluted with water ( $\mathrm{pH} 7.5)$ by a factor of $1: 20$, and analyzed by UV spectroscopy (Thermo Scientific, Model Genesys 10). The second-order derivative of the UV spectra was obtained to determine the amount of drug released. A calibration curve of IB at $\mathrm{pH}$ of 7.5 was used.

\section{Results and Discussion}

3.1. FTIR Spectroscopy. Ibuprofen, unloaded PECA nanoparticles, and PECA nanoparticles loaded with ibuprofen were analyzed by infrared spectroscopy. IR spectra of pure PECA (a), ibuprofen (b), and PECA loaded with IB (c) are shown in Figure 1. In the IR spectrum of ibuprofen, its characteristic 


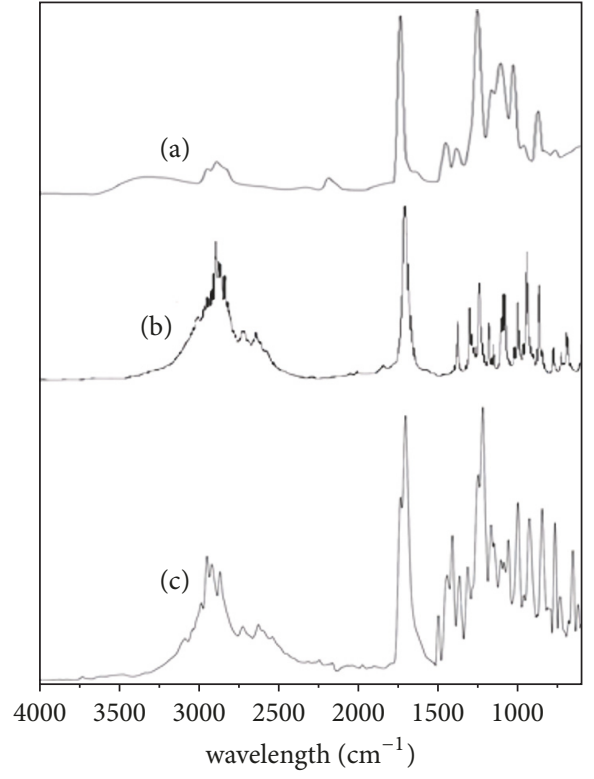

FIgURE 1: IR spectra of (a) PECA, (b) ibuprofen, and (c) PECA loaded with IB.

drug signals are observed: between 2800 and $3200 \mathrm{~cm}^{-1}$ a broadband due to stretching bands of the methyl, methylene, and $\mathrm{C}-\mathrm{H}$ aromatic groups, at $1715 \mathrm{~cm}^{-1}$ a band due to the vibration mode of the carbonyl group, and finally, a group of signals between 750 and $900 \mathrm{~cm}^{-1}$. In the spectrum of the PECA loaded with IB, the characteristic bands of the drug can be observed: the broad band between 2800 and $3200 \mathrm{~cm}^{-1}$, the signals between 750 and $900 \mathrm{~cm}^{-1}$, and the band at $1715 \mathrm{~cm}^{-1}$ of the carbonyl group at one side of the carbonyl group band of the PECA $\left(1750 \mathrm{~cm}^{-1}\right)$. Similar spectra were obtained for the other PECA nanoparticles prepared in the presence of IB, which confirms that the polymer was charged with the drug.

3.2. Molecular Weight. Number-average molecular weights of the PECA obtained from the polymerizations in the presence of IB are relatively low, varying between 3,500 and $4,600 \mathrm{~g} / \mathrm{mol}$ with low polydispersity (around 1.05). These values are on the order of magnitude reported for the polymerization of ethyl cyanoacrylate by anionic polymerization in aqueous dispersions stabilized with surfactants [21-23]. The low molecular weights obtained in these polymerizations are due to a high termination rate of growing chains as a consequence of the large availability of $\mathrm{H}^{+}$when the reaction is carried out at low $\mathrm{pH}$. A more detailed explanation on this subject has been reported elsewhere by our group [13].

3.3. Particle Size and IB Loading Efficiency. Polymerizations with and without IB were carried out at $\mathrm{pH}$ of $1.75,15^{\circ} \mathrm{C}$, $0.08 \mathrm{~g}$ of TW80, and $1 \mathrm{~g}$ of ECA. The Dz of the nanoparticles without IB, obtained by QLS, was $108 \mathrm{~nm}$. A representative micrograph of the PECA nanoparticles without IB is shown in Figure 2, where some particle agglomeration can be observed; a particle size distribution histogram is presented

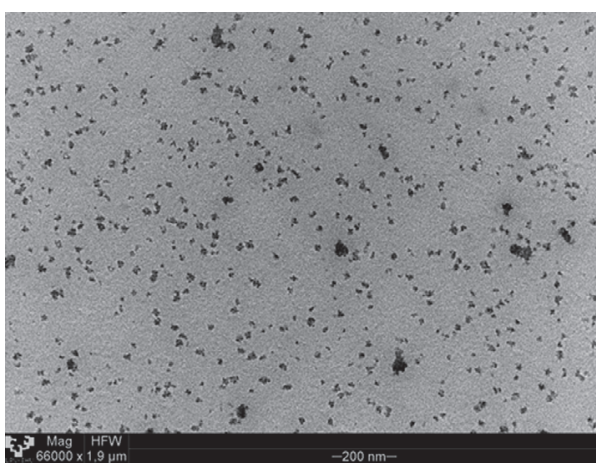

FIGURE 2: Micrography of particles obtained at $\mathrm{pH}$ of $1.75,15^{\circ} \mathrm{C}$, $0.08 \mathrm{~g}$ of TW80, and $1 \mathrm{~g}$ of ECA.

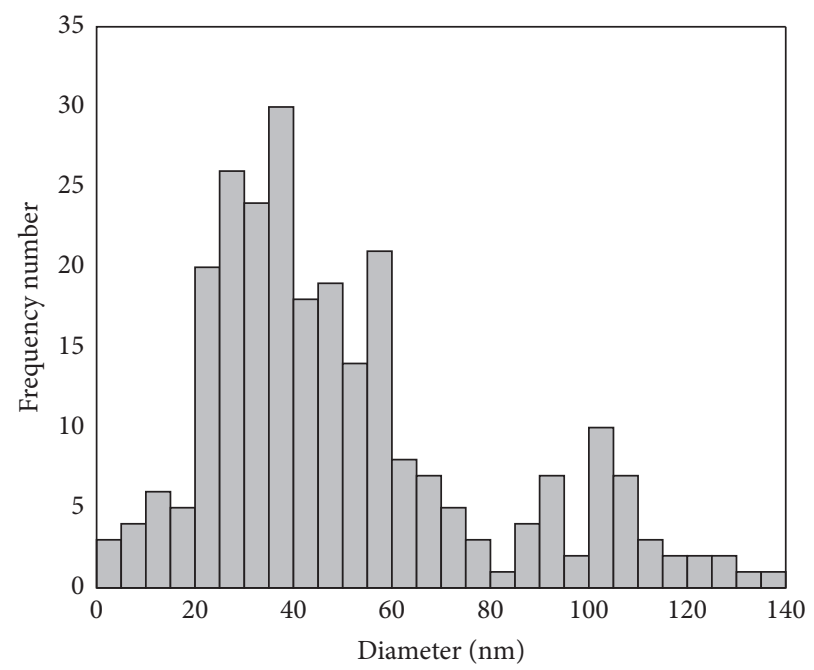

FIgURE 3: Histograms of the particles obtained at $\mathrm{pH}$ of $1.75,15^{\circ} \mathrm{C}$, $0.08 \mathrm{~g}$ of TW80, and $1 \mathrm{~g}$ of ECA.

in Figure 3, summarizing measurements of over 300 particles. The histogram indicates that the particles have a bimodal distribution. The diameter of the smaller particles ranges from 70 to $80 \mathrm{~nm}$, while the larger particles have sizes in the range of 80 to $140 \mathrm{~nm}$. The PECA nanoparticles in Figure 4 correspond to those obtained at $\mathrm{pH}$ of $1.75,15^{\circ} \mathrm{C}, 0.08 \mathrm{~g}$ of TW80, and $1 \mathrm{~g}$ of ECA in the presence of IB $(0.25 \mathrm{~g})$ and have smaller $\mathrm{Dz}(55 \mathrm{~nm})$ than that obtained without IB; in addition, the particle distribution obtained from TEM shows only one population (Figure 5), whereas the polymerization of ECA under the same conditions but without ibuprofen shows a bimodal distribution (Figure 3 ). The Dn value from TEM for the sample from the polymerization with IB was $56 \mathrm{~nm}$, which matches very well with its $\mathrm{Dz}$ value of $55 \mathrm{~nm}$ from the QLS measurement. Then, it is evident that the presence of IB reduces the final particle size. In the polymerization performed without IB, the population of larger particles would be the result of coalescence of a fraction of the smaller particles in order to reduce the total interface area to obtain a more stable latex [24]. However, in the presence of IB, there is only one population, which could be attributed to the 
TABLE 1: Average particle size and IB content of the nanoparticles.

\begin{tabular}{lcccccc}
\hline Polymerization & ECA $(\mathrm{g})$ & TW80 $(\mathrm{g})$ & $\mathrm{pH}$ & $\mathrm{T}\left({ }^{\circ} \mathrm{C}\right)$ & Loading efficiency $(\%)$ & $\mathrm{Dz}^{1}(\mathrm{~nm})$ \\
\hline F1/5/1 & 1 & 0.16 & 1 & 5 & $26.4 \pm 1.4$ & $58.1 \pm 2.3$ \\
F1/5/1.5 & 1 & 0.16 & 1.5 & 5 & $17.5 \pm 1.3$ & $55.3 \pm 1.7$ \\
F.5/15/15 & 0.5 & 0.08 & 1 & 15 & $26.4 \pm 1.2$ & $42.9 \pm 1.8$ \\
F.5/15/1.5 & 0.5 & 0.08 & 1.5 & 15 & $30.8 \pm 0.7$ \\
F.5/15/1.75 & 0.5 & 0.08 & 1.75 & 15 & $1.2 \pm 2.1$ \\
\hline
\end{tabular}

${ }^{1}$ Determined by QLS.

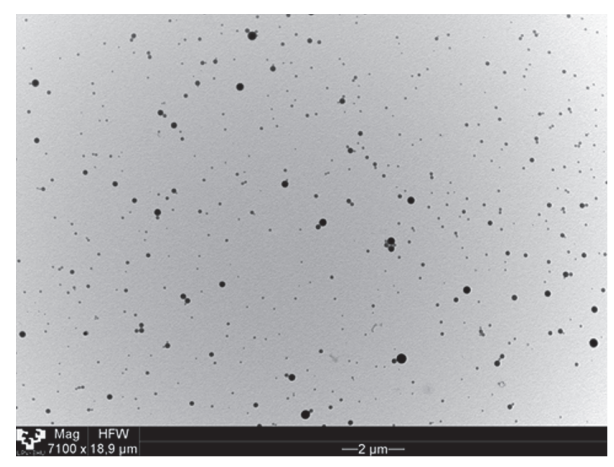

Figure 4: Micrography of particles obtained at $\mathrm{pH}$ of $1.75,15^{\circ} \mathrm{C}$, $0.08 \mathrm{~g}$ of TW80, $1 \mathrm{~g}$ of ECA, and $0.25 \mathrm{~g}$ of IB.

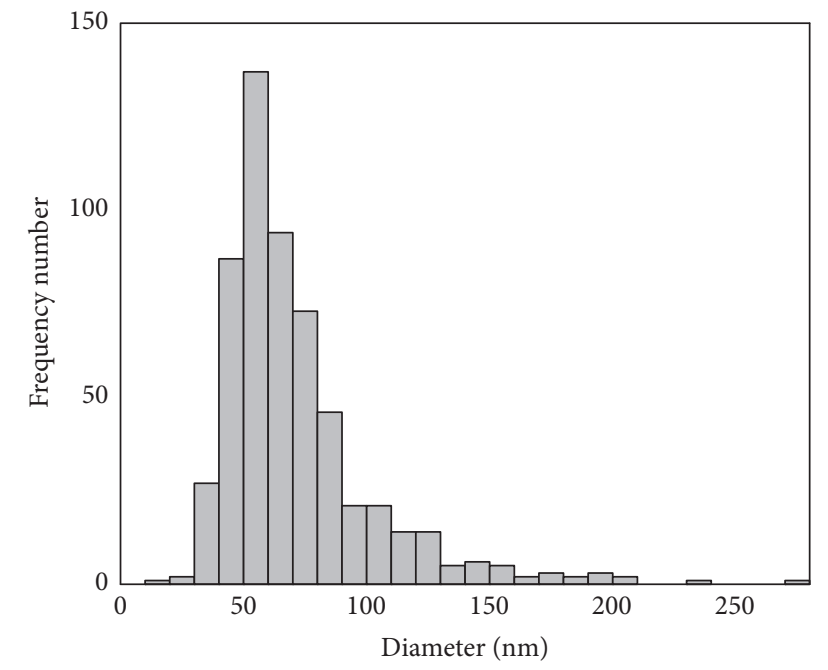

Figure 5: Histogram of the particles obtained at $\mathrm{pH}$ of $1.75,15^{\circ} \mathrm{C}$, $0.08 \mathrm{~g}$ of TW80, $1 \mathrm{~g}$ of ECA, and $0.25 \mathrm{~g}$ of IB.

IB molecules near the particle surface acting as a protective agent against coalescence.

Table 1 shows the particle sizes (Dz) obtained in the polymerizations carried out in the presence of $I B$, varying the TW80 content, $\mathrm{pH}$, and temperature. Particle sizes from 10 to $58 \mathrm{~nm}$ were obtained depending on the reaction conditions. Moreover, IB-loaded nanoparticles so small as $10 \mathrm{~nm}$ in $\mathrm{Dz}$ were obtained when the polymerization was performed at $\mathrm{pH}$ 1.75 .

The data in Table 1 indicates that the particle size has an inverse dependence on $\mathrm{pH}$, which is more evident in the polymerizations performed at $15^{\circ} \mathrm{C}$. This behavior would arise from an increase in the number of hydroxyl ions as the $\mathrm{pH}$ increases. As is well known, the mechanism of anionic polymerization of alkyl cyanoacrylates in aqueous dispersions containing micelles includes an initiation via hydroxyl ions in the aqueous phase, which react with the monomer near the swollen micelles surface, giving rise to a particle [25]. The inverse dependence of particle size on $\mathrm{pH}$ has been previously reported in the specialized literature [24-26].

A more careful analysis needs to be carried out when comparing the particle sizes of the polymerizations conducted at different temperature and ECA concentration (F1/5/1 versus F.5/15/1). Reports in the specialized literature on the effect of temperature on particle size in anionic polymerization of alkyl cyanoacrylates in aqueous dispersions containing surfactants are scarce. Douglas et al. [26] polymerized butyl-2-cyanoacrylate using Dextran 70 as the surfactant, finding a slight increase in $\mathrm{Dz}$ with temperature in the $4-80^{\circ} \mathrm{C}$ interval. This effect is ascribed by the authors to an increase in particle coagulation caused by the rise in the kinetic energy of the system. More recently, Yang et al. [27] also documented a slight increase in particle size in the range $10-50^{\circ} \mathrm{C}$ in the polymerization of butyl cyanoacrylate using chitosan as the stabilizer. No explanation on this effect was offered by the authors. In accordance with these reports, an increase in polymerization temperature would lead to larger particles, but the decrease in monomer concentration, at the same surfactant content, would cause a diminution in $\mathrm{Dz}$, because more surfactant molecules are available for particle stabilization. Table 1 shows that a smaller $\mathrm{Dz}$ was obtained when the polymerization was carried out at $15^{\circ} \mathrm{C}$ and $0.5 \mathrm{~g}$ of ECA, whereby the effect on the particle size of the decrease in monomer concentration predominated over that of the increase in temperature.

Particle size is an important feature for nanostructures to be used as platform for drug delivery. As mentioned above, particles with diameters just above $5.5 \mathrm{~nm}$ would remain for long periods in the blood stream because they would be able to avoid renal removal, as well as their increased probability for immunological system evasion. To the best of our knowledge, there are no reports in the specialized literature on the preparation of PECA nanoparticles with $10 \mathrm{~nm}$ in mean diameter and even less, such small PECA nanoparticles containing IB; there is only one report documenting the obtaining of so small drug-loaded polymeric nanoparticles [28]. This report, also from our group, deals with the preparation of nanoparticles composed of poly(methyl-methacrylateco-methacrylic acid), 2/1 ( $\mathrm{mol} / \mathrm{mol})$, a biocompatible and 
TABLE 2: $R^{2}$ values for the drug release models used to fit the experimental data.

\begin{tabular}{lccccc}
\hline Model & & \multicolumn{3}{c}{ Experimental conditions } \\
& F1/5/1 & F1/5/1.5 & F.5/15/1 & F.5/15/1.5 & 0.77 \\
Higuchi & 0.94 & 0.93 & 0.70 & 0.87 & 0.90 \\
Hixson & 0.96 & 0.98 & 0.85 & 0.94 & 0.94 \\
Korsmeyer-Peppas & 0.98 & 0.97 & 0.96 & 0.98 \\
Weibull & 0.99 & 0.99 & 0.97 & 0.98 \\
\hline
\end{tabular}

biodegradable material approved by the FDA, loaded with IB.

Table 1 also shows that drug loading efficiency varies from 17.6 to $30.8 \%$ depending on the reaction conditions. Looking at Table 1 , it would seem that higher $\mathrm{pH}$ favors a greater IB loading efficiency. This makes sense because, at the $\mathrm{pH}$ used to synthetize the nanoparticles, the IB is poorly soluble in water preferring to be inside the micelles, which become nanoparticles when the polymerization takes place. As the $\mathrm{pH}$ increases, more particles are formed, resulting in more IB being trapped into the PECA nanoparticles.

3.4. In Vitro Release Study. UV spectroscopy was used to determine the amount of drug released. The wavelength that has been employed to determine the presence of ibuprofen is $264 \mathrm{~nm}$ [29]. However, the absorption bands of PECA and IB overlap at that wavelength. For this reason, the determinations were done at $\mathrm{pH}$ of 7.5 and a wavelength of $220 \mathrm{~nm}$ [30], using the second derivative of the spectra to obtain more accurate values [31].

The models used to fit the experimental release data were those of Higuchi (1); Korsmeyer-Peppas (2); Hixson (3); and Weibull (4).

$$
\begin{aligned}
M & =k_{H} \sqrt{t}, \\
\frac{M}{M_{0}} & =k_{1} t^{n}, \\
k_{3} t & =M_{0}^{1 / 3}-M^{1 / 3}, \\
\frac{M}{M_{0}} & =\left(1-e^{-t / k_{w}}\right)^{n},
\end{aligned}
$$

where $M$ is the amount of drug released at time $t ; M_{0}$ is the initial amount of drug in the particles; $k_{H}$ is the Higuchi dissolution constant; in the Korsmeyer-Peppas equation, $k_{1}$ is a constant; and $n$ indicates, according to its value, the release mechanism; $k_{3}$ is a constant in the Hixson equation; and $k_{w}$ is the Weibull constant related to the inverse of the time necessary to release $63.2 \%$ of the drug, while the value of $n$ indicates the transport mechanism [32].

The experimental data from IB release runs were fitted using the program Origin Pro 8. Table 2 shows the values of the constants of the drug release and the $R^{2}$ values corresponding to the best fit for each model. Analyzing the values of $R^{2}$, it was found that their higher values correspond to the Weibull model. However, statistically it is not possible to rule out the Korsmeyer-Peppas and Hixson models. Our

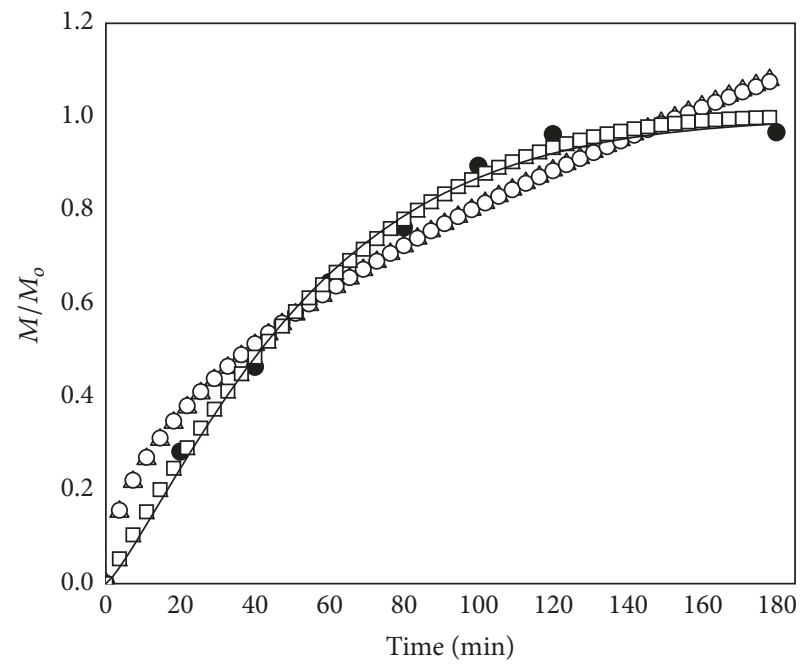

FIGURE 6: Curve fitting to the release of ibuprofen from nanoparticles prepared at F1/5/1 conditions: equations of $(\triangle)$ Higuchi, $(O)$ Korsmeyer-Peppas, and ( $\square$ ) Hixson; the line represents Weibull equation and $(\bullet)$ the experimental data.

results rule out the Higuchi model because the $R^{2}$ values are low.

Figures 6 and 7 depict the drug release profiles from the IB-loaded nanoparticles obtained at two reaction conditions (F1/5/1 and F.5/15/1.75). In accordance with the experimental data, it would appear that the release starts at a low rate; however most of the IB is released within the first 80-120 min. Figures 6 and 7 include the four models used here to fit experimental data, which suggest that the Weibull model is the one that best fits those data. The Weibull model also was the one that best fits the release profiles of the IB-loaded nanoparticles obtained in the other experiments. Although the most used models to predict drug release kinetics employed in the literature are those of Higuchi and Korsmeyer-Peppas because they usually give a good fit to the kinetic release data, the models predict that $M / M_{0}$ always increases. Then at sufficiently long release times $M / M_{0}$ becomes greater than 1, which does not make sense since it is not possible to release more drug than it was loaded.

Deriving the theoretical release curves obtained from the best fit to the Korsmeyer-Peppas, Hixson, and Weibull models, we obtained the release rates as a function of time. Figure 8 shows the drug release rate curves for the different models as a function of time for the F1/5/1.5 sample. A similar drug release behavior was obtained for the other IB-loaded 


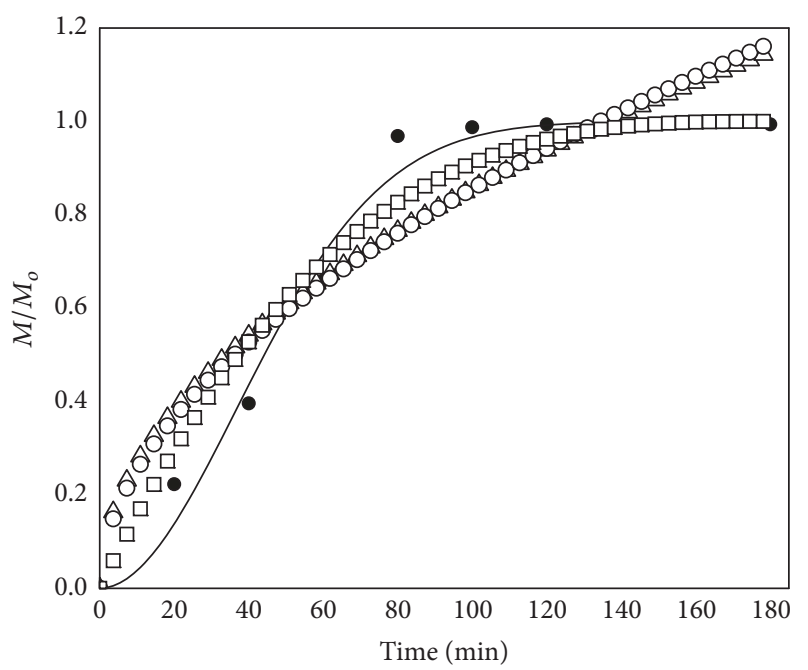

FIGURE 7: Curve fitting to the release of ibuprofen from nanoparticles prepared at F.5/15/1.75 conditions: equations of $(\triangle)$ Higuchi, (○) Korsmeyer-Peppas, and ( $\square$ ) Hixson; the line represents Weibull equation and $(\bullet)$ the experimental data.

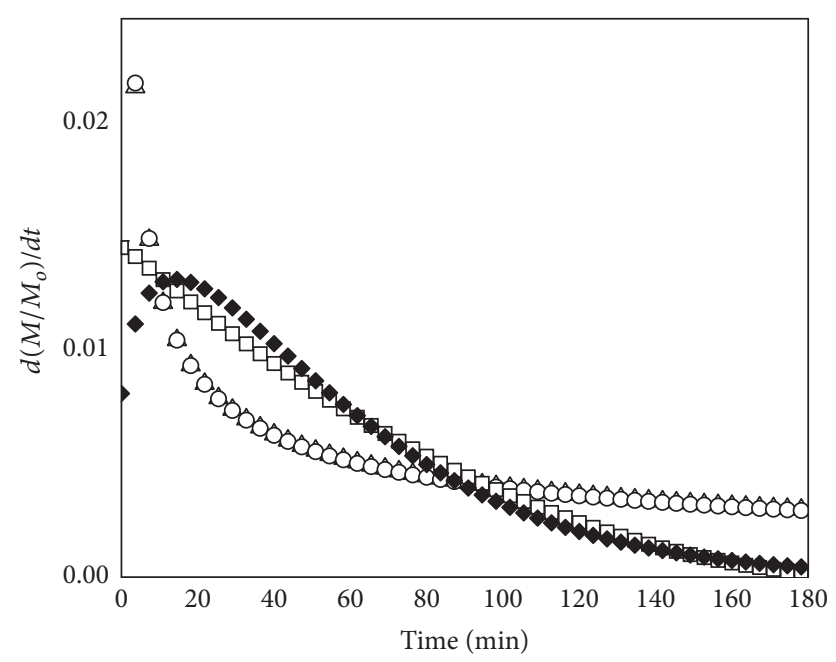

FIgURE 8: Release rate for sample F1/5/1: models of $(\triangle)$ Higuchi, $(\bigcirc)$ Korsmeyer-Peppas, ( $\square$ ) Hixson, and $(\diamond)$ Weibull.

nanoparticles. As can be seen in Figure 8, with the exception of the Weibull model, the other models predict that initially there is a significant amount of drug released (high release rate) and that as time passes the released amount decreases. Since the experimental data show that initially the release rate is slow, followed by its increase and its eventual decline, the only model able to adjust to the experimental data is that of Weibull, a model that has been widely applied to drug release analysis [29, 33-36]. Furthermore, Papadopoulou et al. [34] demonstrated that the exponent $n$ of the Weibull function is an indicator of the mechanism of transport of the drug through the polymer matrix. Values of $n \leq 0.75$ indicate Fick diffusion in either fractal or Euclidean spaces, whereas a combined mechanism (Fick diffusion and transport case II) is associated with values in the range $0.75<n<1$. For values of
TABLE 3: $k$ and $n$ values in Weibull equation for ibuprofen release from nanoparticles.

\begin{tabular}{lcc}
\hline Sample & $k$ & $n$ \\
\hline F1/5/1 & 0.47 & 1.37 \\
F1/5/1.5 & 0.62 & 1.20 \\
F.5/15/1 & 0.79 & 2.44 \\
F.5/15/1.5 & 0.68 & 1.86 \\
F.5/15/1.75 & 0.44 & 1.94 \\
\hline
\end{tabular}

$n$ greater than 1 , drug transport follows a complex mechanism of release [34], and the shape of the curve becomes sigmoidal.

Table 3 shows that for the Weibull model in all cases the exponent $n$ is higher than 1 , which agrees well with the sigmoidal behavior of the drug release from the PECA nanoparticles. The relatively slow release rate at the beginning of the process could be attributed to the start of the IB dissolution process; then the release rate becomes faster as the water penetrates the particles and more drug is dissolved; finally, as a greater amount of drug is released, its concentration within the particles decreases causing a reduction in the IB release. Table 3 also includes the value of $k$ in the Weibull equation, which is inversely proportional to the time interval necessary to release $63.2 \%$ of the drug [32].

\section{Conclusions}

Semicontinuous heterophase polymerization allowed the preparation of IB-loaded PECA nanoparticles with mean diameters between 10 and $58 \mathrm{~nm}$. To our best knowledge, this is the first report on the preparation of drug-loaded PECA nanoparticles with a mean diameter as small as $10 \mathrm{~nm}$. Of the variables considered in this study, the $\mathrm{pH}$ is the one that mainly determines the size of the loaded nanoparticles, as well as the drug loading efficiency; higher $\mathrm{pH}$ results in smaller particles and a larger amount of IB loaded. The analysis of the IB release tests indicated that the Weibull model is the one that best fits the experimental data. In addition, it was found that most of the IB is released within the first $80-120$ min but starts at a slow rate, which contrasts with the burst of the drug concentration in the plasma when IB is administrated orally.

\section{Conflicts of Interest}

No potential conflicts of interest were reported by the authors.

\section{Acknowledgments}

National Council of Science and Technology (CONACyT) supported this research through Grant 2014-223227. One of the authors (J. A. Balleño) acknowledges the scholarship from CONACyT.

\section{References}

[1] V. Sanna, N. Pala, and M. Sechi, "Targeted therapy using nanotechnology: focus on cancer," International Journal of Nanomedicine, vol. 9, no. 1, pp. 467-483, 2014. 
[2] C. I. C. Crucho, "Stimuli-responsive polymeric nanoparticles for nanomedicine," ChemMedChem, vol. 10, no. 1, pp. 24-38, 2015.

[3] Q.-H. Zhao and L.-Y. Qiu, "An overview of the pharmacokinetics of polymer-based nanoassemblies and nanoparticles," Current Drug Metabolism, vol. 14, no. 8, pp. 832-839, 2013.

[4] R. Sunasee, C. K. Adokoh, J. Darkwa, and R. Narain, “Therapeutic potential of carbohydrate-based polymeric and nanoparticle systems," Expert Opinion on Drug Delivery, vol. 11, no. 6, pp. 867-884, 2014.

[5] J. L. Markman, A. Rekechenetskiy, E. Holler, and J. Y. Ljubimova, "Nanomedicine therapeutic approaches to overcome cancer drug resistance," Advanced Drug Delivery Reviews, vol. 65, no. 13-14, pp. 1866-1879, 2013.

[6] N. Kamaly, Z. Xiao, P. M. Valencia, A. F. Radovic-Moreno, and O. C. Farokhzad, "Targeted polymeric therapeutic nanoparticles: Design, development and clinical translation," Chemical Society Reviews, vol. 41, no. 7, pp. 2971-3010, 2012.

[7] S. R. D’Mello, S. K. Das, and N. G. Das, "Drug delivery nanoparticles formulation and characterization," Informa Healthcare USA, Inc., New York, NY, USA. 2009.

[8] J. Liu, M. Yu, X. Ning, C. Zhou, S. Yang, and J. Zheng, "PEGylation and zwitterionization: Pros and cons in the renal clearance and tumor targeting of near-IR-emitting gold nanoparticles," Angewandte Chemie International Edition, vol. 52, no. 48, pp. 12572-12576, 2013.

[9] S. M. Moghimi, C. J. H. Porter, I. S. Muir, L. Illum, and S. S. Davis, "Non-phagocytic uptake of intravenously injected microspheres in rat spleen: Influence of particle size and hydrophilic coating," Biochemical and Biophysical Research Communications, vol. 177, no. 2, pp. 861-866, 1991.

[10] T. Kissel and M. Roser, "Influence of chemical surface-modification on the phagocytic properties of albumin nanoparticles," Proceedings International Symposium Controlled Release Bioactive Materials, vol. 18, pp. 275-276, 1991.

[11] A. Albanese, P. S. Tang, and W. C. W. Chan, "The effect of nanoparticle size, shape, and surface chemistry on biological systems," Annual Review of Biomedical Engineering, vol. 14, pp. $1-16,2012$.

[12] M. Elsabahy and K. L. Wooley, "Design of polymeric nanoparticles for biomedical delivery applications," Chemical Society Reviews, vol. 41, no. 7, pp. 2545-2561, 2012.

[13] H. Saade, C. Barrera, G. Guerrero, E. Mendizábal, J. E. Puig, and R. G. López, "Preparation and loaded with rifampicin of sub$50 \mathrm{~nm}$ poly(ethyl cyanoacrylate)," Journal of Nanomaterials, vol. 2016, Article ID 384973, 11 pages, 2016.

[14] R. Ledezma, M. E. Treviño, L. E. Elizalde et al., "Semicontinuous heterophase polymerization under monomer starved conditions to prepare nanoparticles with narrow size distribution," Journal of Polymer Science Part A: Polymer Chemistry, vol. 45, no. 8, pp. 1463-1473, 2007.

[15] W. V. Smith and R. H. Ewart, "Kinetics of emulsion polymerization," The Journal of Chemical Physics, vol. 16, no. 6, pp. 592-599, 1948.

[16] J. J. Krackeler and H. Naidus, "Particle size and molecular weight distributions of various polystyrene emulsions," Journal of Polymer Science C: Polymer Letters, vol. 27, no. 1, pp. 207-235, 1969.

[17] H. Saade, C. Barrera, A. Espinoza, M. L. Lopez-Quintanilla, S. Fernandez, and R. G. Lopez, "Ultrafine Nanoparticles of
Ibuprofen-Poly(methyl methacrylate) by a PolymerizationLoading Method," Drug Delivery Letters, vol. 3, no. 1, pp. 54-60, 2013.

[18] H. Saade, J. C. Garza, M. L. Lopez-Quintanilla et al., "Semicontinuous heterophase polymerization as a tool for preparing ultrafine nanoparticles of ibuprofen-poly(methyl methacrylate)," Nano, vol. 9, no. 6, Article ID 1450060, 2014.

[19] E. I. G. García, M. E. G. Alegre, M. A. C. Sánchez, and A. I. T. Suárez, "Estudio de solubilidad de ibuprofeno en medio acuoso: elaboración de una formulación líquida de uso pediátrico," In VI Congreso SEFIG; 201AD, pp. 161-164.

[20] L. R. Shaw, W. J. Irwin, T. J. Grattan, and B. R. Conway, “The effect of selected water-soluble excipients on the dissolution of paracetamol and ibuprofen," Drug Development and Industrial Pharmacy, vol. 31, no. 6, pp. 515-525, 2005.

[21] M. G. Han, S. Kim, and S. X. Liu, "Synthesis and degradation behavior of poly(ethyl cyanoacrylate)," Polymer Degradation and Stability, vol. 93, no. 7, pp. 1243-1251, 2008.

[22] B. Seijo, E. Fattal, L. Roblot-Treupel, and P. Couvreur, "Design of nanoparticles of less than $50 \mathrm{~nm}$ diameter: preparation, characterization and drug loading," International Journal of Pharmaceutics, vol. 62, no. 1, pp. 1-7, 1990.

[23] L. Z. Zhaparova, Y. M. Tazhbayev, M. Z. Burkeev et al., "Synthesis and characterization of polyethyl cyanoacrylate nanoparticles loaded with capreomycin sulfate," Pharmaceutical Chemistry Journal, vol. 46, no. 1, pp. 6-9, 2012.

[24] H. Saade, S. Torres, C. Barrera, J. Sánchez, Y. Garza, and R. G. López, "Effect of $\mathrm{pH}$ and monomer dosing rate in the anionic polymerization of ethyl cyanoacrylate in semicontinuous operation," International Journal of Polymer Science, vol. 2015, Article ID 827059, 9 pages, 2015.

[25] N. Behan, C. Birkinshaw, and N. Clarke, "Poly n-butyl cyanoacrylate nanoparticles: a mechanistic study of polymerisation and particle formation," Biomaterials, vol. 22, no. 11, pp. 1335-1344, 2001.

[26] S. J. Douglas, L. Illum, S. S. Davis, and J. Krueter, "Particle size and size distribution of poly(butyl-2-cyanoacrylate) nanoparticles. I. Influence of physicochemical factors," Journal of Colloid and Interface Science, vol. 101, no. 1, pp. 149-158, 1984.

[27] S. C. Yang, H. X. Ge, Y. Hu, X. Q. Jiang, and C. Z. Yang, "Formation of positively charged poly(butyl cyanoacrylate) nanoparticles stabilized with chitosan," Colloid and Polymer Science, vol. 278, no. 4, pp. 285-292, 2000.

[28] H. Saade, M. L. De Guillén, J. C. Romero et al., "Biocompatible and Biodegradable Ultrafine Nanoparticles of Poly(Methyl Methacrylate-co-Methacrylic Acid) Prepared via Semicontinuous Heterophase Polymerization: Kinetics and Product Characterization," International Journal of Polymer Science, vol. 2016, Article ID 7674620, 2016.

[29] F. O. Costa, J. J. S. Sousa, A. A. C. C. Pais, and S. J. Formosinho, "Comparison of dissolution profiles of Ibuprofen pellets," Journal of Controlled Release, vol. 89, no. 2, pp. 199-212, 2003.

[30] X. W. Teng, S. W. J. Wang, and N. M. Davies, "Stereospecific high-performance liquid chromatographic analysis of ibuprofen in rat serum," Journal of Chromatography B, vol. 796, no. 2, pp. 225-231, 2003.

[31] L. Wang and M. Asgharnejad, "Second-derivative UV spectrometric determination of simvastatin in its tablet dosage form," Journal of Pharmaceutical and Biomedical Analysis, vol. 21, no. 6, pp. 1243-1248, 2000. 
[32] K. H. Ramteke, A. R. Kharat, and S. V. Patil, "Mathematical models of drug dissolution: A review," Scholar Academic Journal of Pharmacy, vol. 3, no. 5, pp. 388-396, 2014.

[33] M. V. S. Varma, A. M. Kaushal, and S. Garg, "Influence of micro-environmental $\mathrm{pH}$ on the gel layer behavior and release of a basic drug from various hydrophilic matrices," Journal of Controlled Release, vol. 103, no. 2, pp. 499-510, 2005.

[34] V. Papadopoulou, K. Kosmidis, M. Vlachou, and P. Macheras, "On the use of the Weibull function for the discernment of drug release mechanisms," International Journal of Pharmaceutics, vol. 309, no. 1-2, pp. 44-50, 2006.

[35] E. Ahnfelt, E. Sjögren, N. Axén, and H. Lennernäs, "A miniaturized in vitro release method for investigating drug-release mechanisms," International Journal of Pharmaceutics, vol. 486, no. 1-2, pp. 339-349, 2015.

[36] L. S. Koester, G. G. Ortega, P. Mayorga, and V. L. Bassani, "Mathematical evaluation of in vitro release profiles of hydroxypropylmethylcellulose matrix tablets containing carbamazepine associated to $\beta$-cyclodextrin," European Journal of Pharmaceutics and Biopharmaceutics, vol. 58, no. 1, pp. 177-179, 2004. 


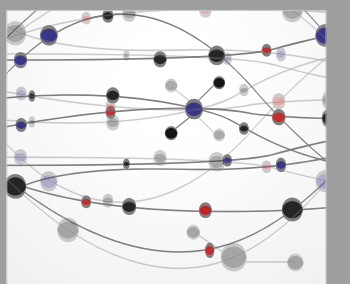

The Scientific World Journal
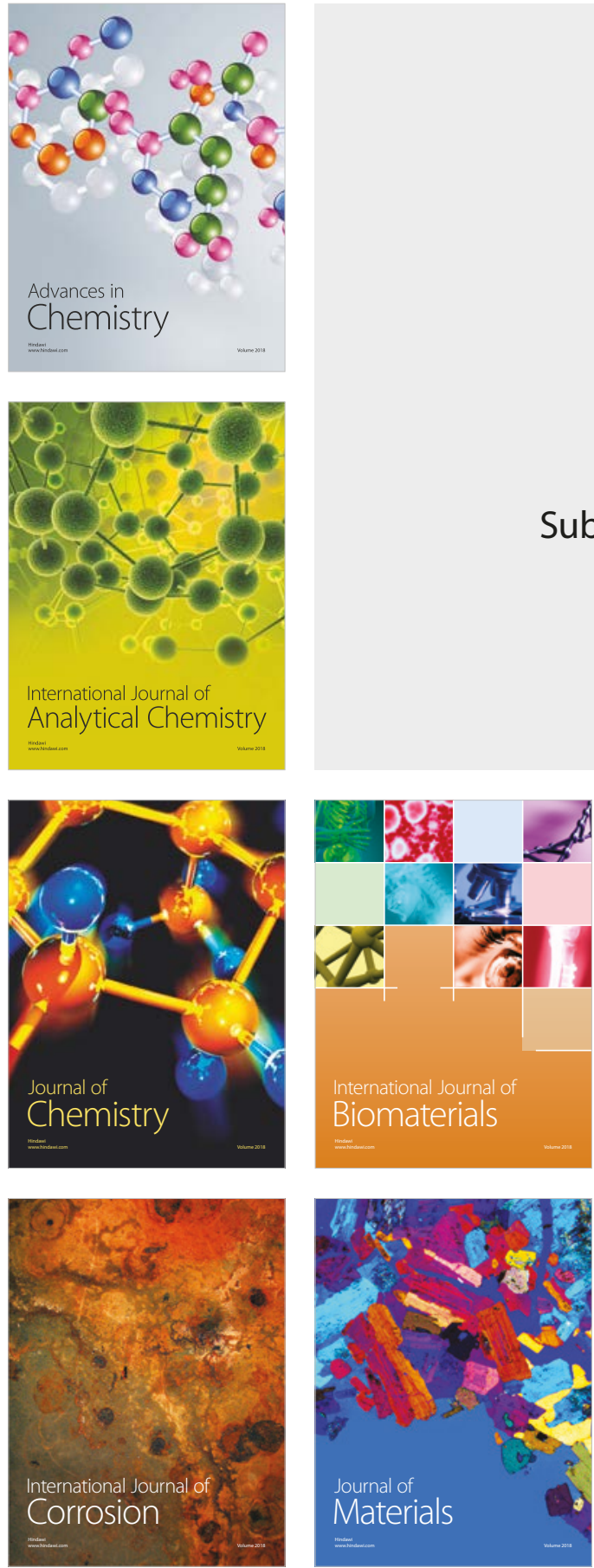

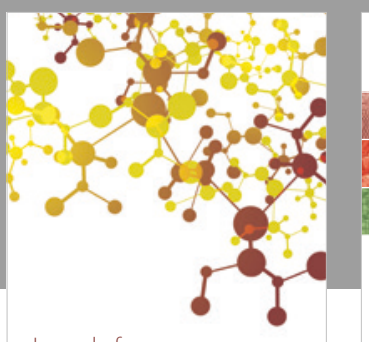

Journal of

Applied Chemistry
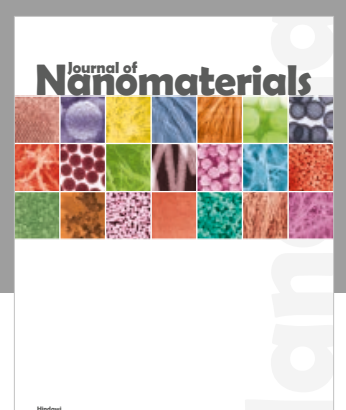

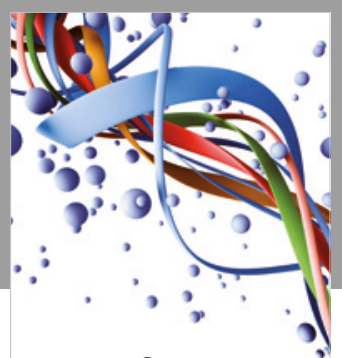

Scientifica

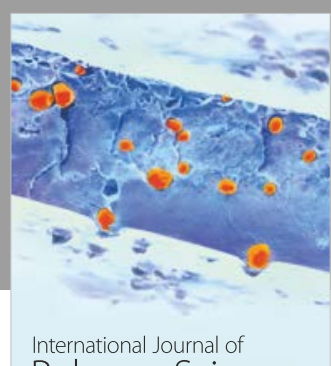

Polymer Science

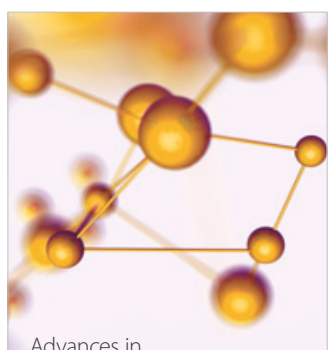

Physical Chemistry
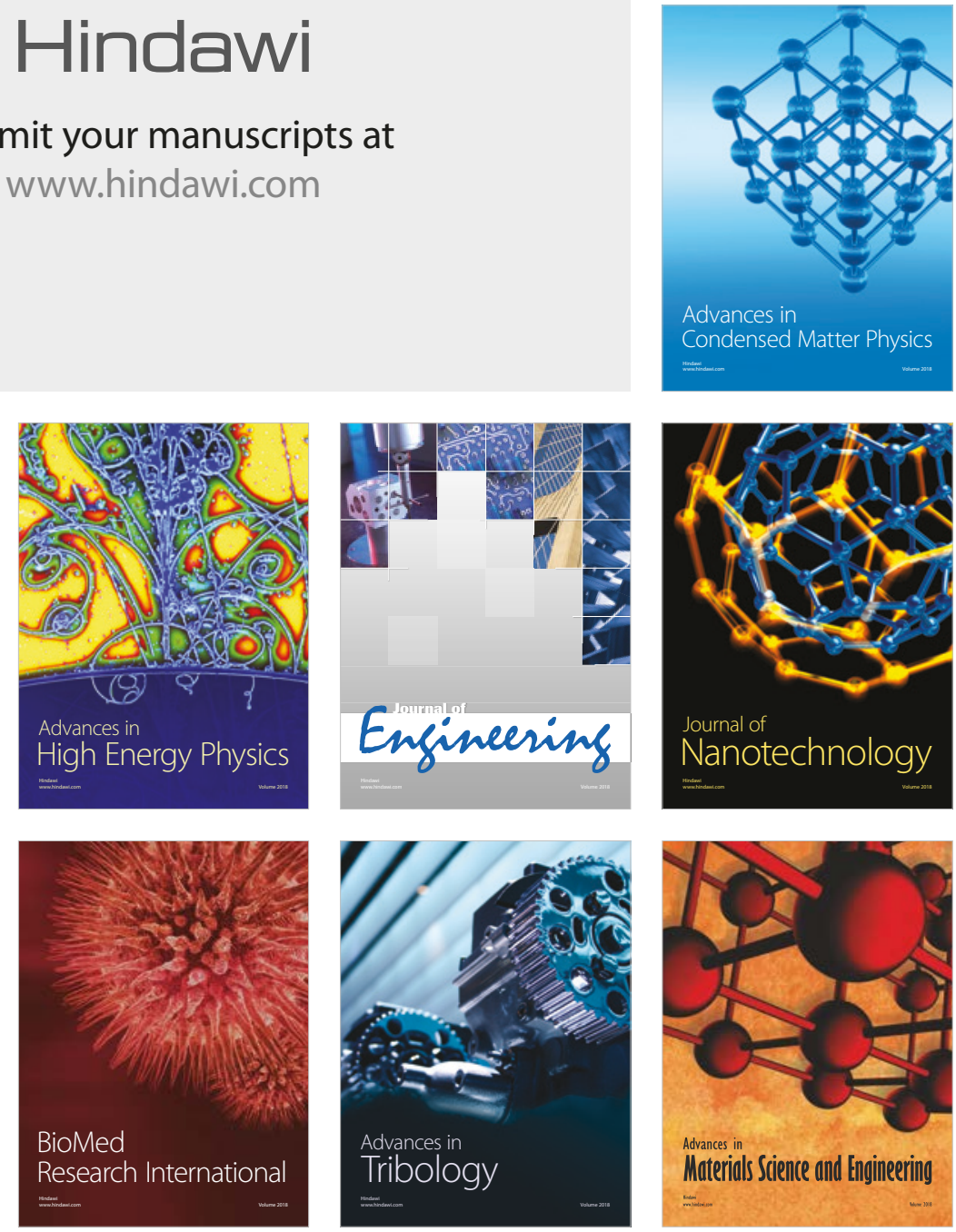